\title{
Realizing an Applicable "Solid $\rightarrow$ Solid" Cathode Process via a Transplantable Solid Electrolyte Interface for Lithium-Sulfur Batteries
}

Xue Chen,,$^{\S}$ Lixia Yuan, ${ }^{*}{ }^{\S}$ Zhen Li, ${ }^{\S}$ Sijing Chen, ${ }^{\ddagger}$ Haijin Ji, ${ }^{\S}$ Yufei Qin, ${ }^{\S}$ Longsheng Wu, ${ }^{*}$

Yue Shen, ${ }^{\S}$ Libin Wang, ${ }^{\S}$ Jingping Hu, ${ }^{*},+$ Yunhui Huang ${ }^{*} \S$

§ State Key Laboratory of Material Processing and Die \& Mould Technology, School of Materials Science and Engineering, Huazhong University of Science and Technology, Wuhan, Hubei 430074, China

${ }^{\sharp}$ School of Environmental Science and Engineering, Huazhong University of Science and Technology, Wuhan, Hubei 430074, China

\section{Corresponding authors}

*E-mail: yuanlixia@hust.edu.cn (L.X.Yuan);

*E-mail: hujp@hust.edu.cn (J.P.Hu);

*E-mail: huangyh@hust.edu.cn (Y.H. Huang). 

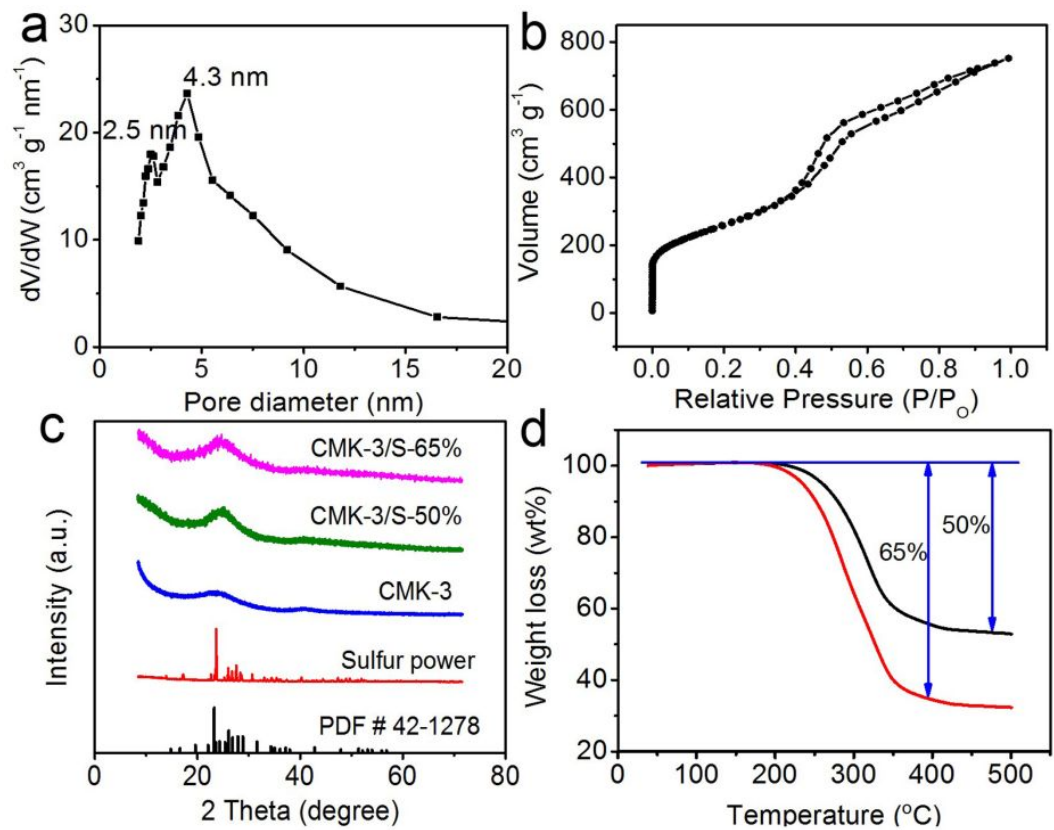

Figure S1. a-b) The pore size distribution a) and nitrogen isothermal adsorption-desorption curve b) of CMK-3, respectively. c) The XRD spectra of S, CMK-3 and CMK-3/S. d) The TG curve of $\mathrm{CMK}-3 / \mathrm{S}$
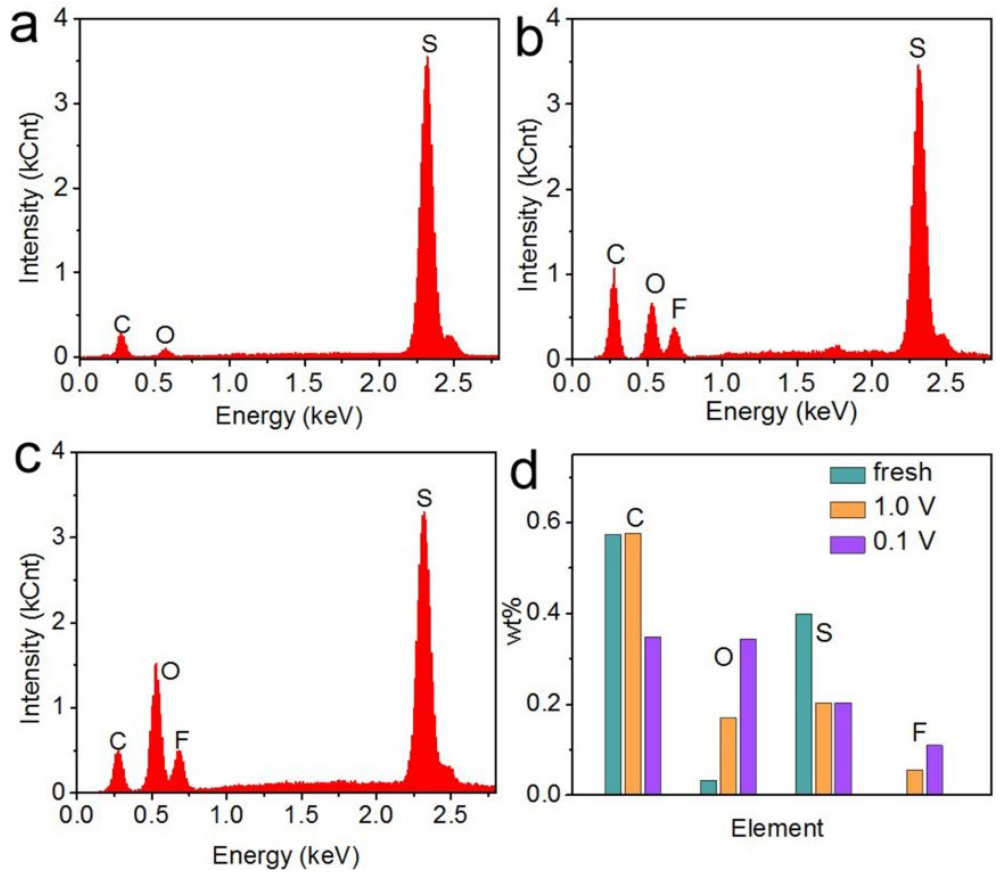

Figure S2. a-c) EDX spectra of the $\mathrm{S}$ cathode at a) fresh, discharged to $1.0 \mathrm{~V} \mathrm{~b}$ ) and $0.1 \mathrm{~V} \mathrm{c}$ ) in $\mathrm{T}_{4.0} \mathrm{~F}_{2.5} \mathrm{C}$ electrolyte. d) The corresponding element mass percentage at different states. 


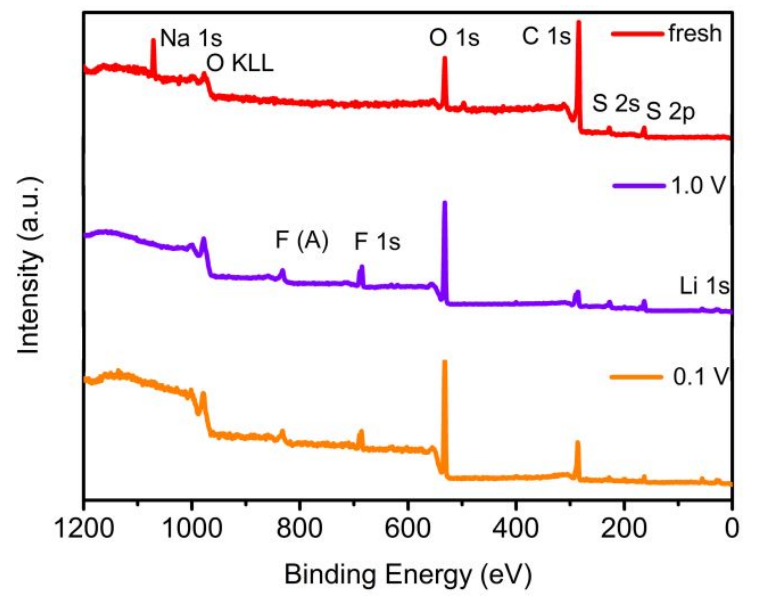

Figure S3. The XPS spectra of CMK-3/S at fresh, discharged to $1.0 \mathrm{~V}$ and $0.1 \mathrm{~V}$ in $\mathrm{T}_{4.0} \mathrm{~F}_{2.5} \mathrm{C}$ electrolyte.
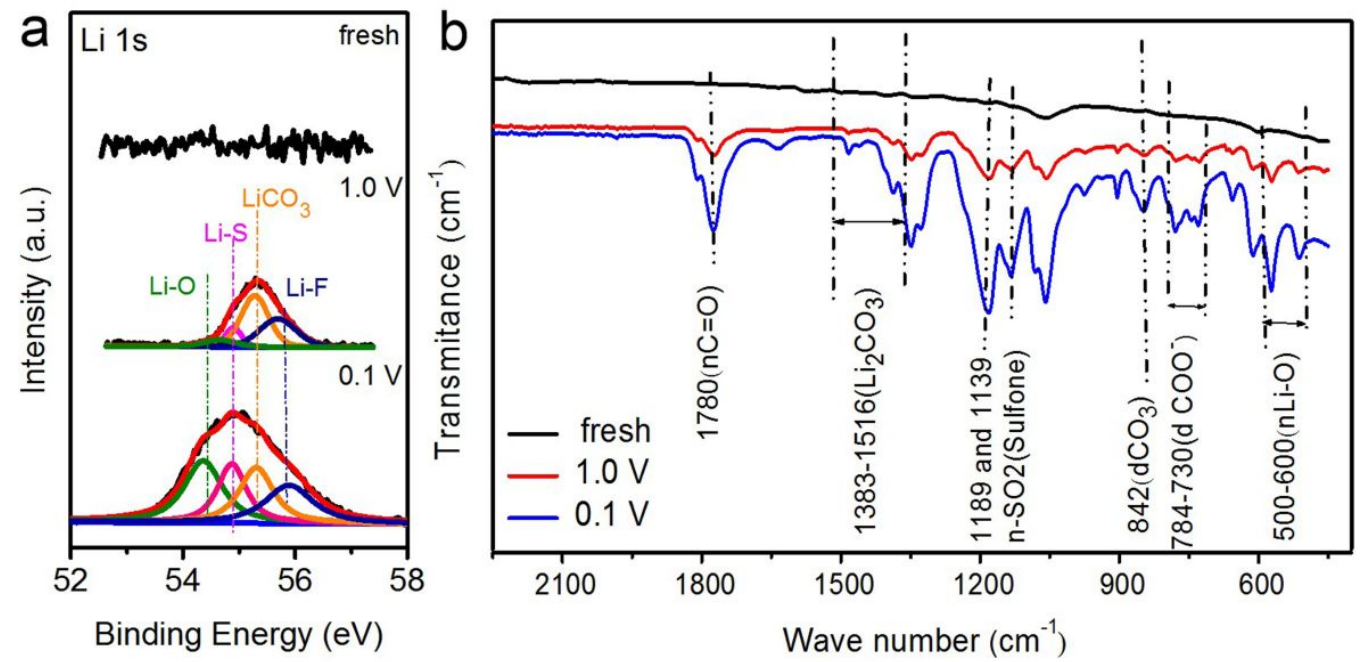

Figure S4. The XPS a) and the ATR-FTIR spectra b) of S cathode at different discharge states (fresh, discharge to $1.0 \mathrm{~V}$ and $0.1 \mathrm{~V}$ in $\mathrm{T}_{4.0} \mathrm{~F}_{2.5} \mathrm{C}$ electrolyte) were shown in: a) Li $1 \mathrm{~s}, \mathrm{~b}$ ), respectively. 
a

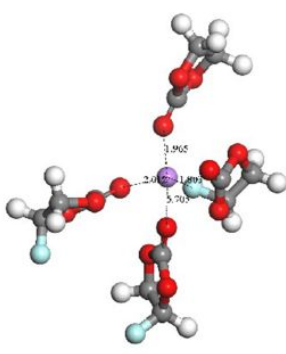

Before geometry optiomization

$\left(\mathrm{T}_{0.5} \mathrm{~F}_{0.5} \mathrm{C}\right)$

b

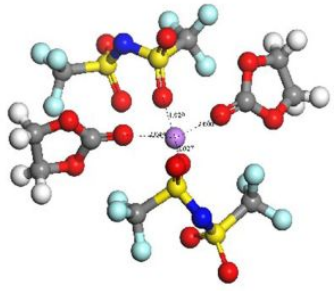

Before geometry optiomization

$\left(\mathrm{T}_{4.0} \mathrm{~F}_{2.5} \mathrm{C}\right)$

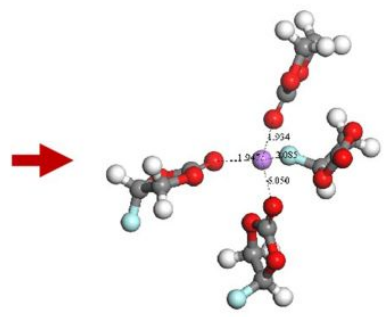

After geometry optimization

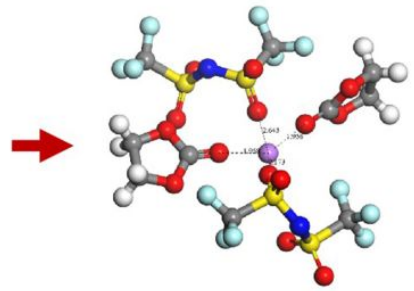

After geometry optimization

Figure S5. Supercells used, structure before and after geometry optimization of a) $\mathrm{T}_{0.5} \mathrm{~F}_{0.5} \mathrm{C}$ and b) $\mathrm{T}_{4.0} \mathrm{~F}_{2.5} \mathrm{C}$.
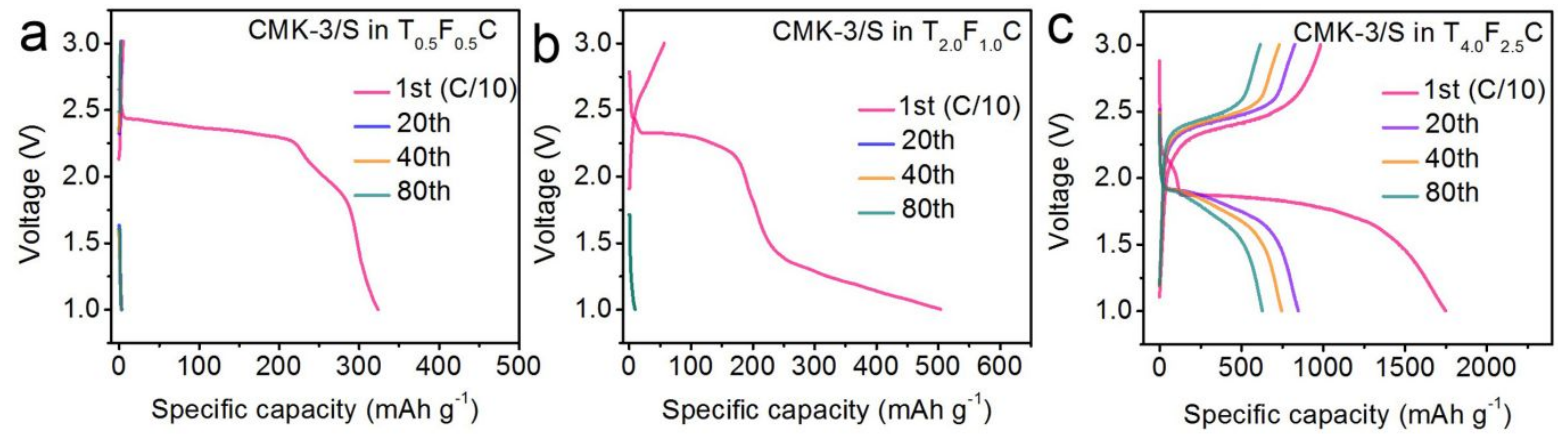

Figure S6. The charge/discharge curves of CMK-3/S in: a) $\mathrm{T}_{0.5} \mathrm{~F}_{0.5}$, b) $\mathrm{T}_{2.0} \mathrm{~F}_{1.0} \mathrm{C}$, c) $\mathrm{T}_{4.0} \mathrm{~F}_{2.5} \mathrm{C}$ with the voltage window is $1.0-3.0 \mathrm{~V}$. Although $\mathrm{CMK}-3 / \mathrm{S}$ can cycle in $\mathrm{T}_{4.0} \mathrm{~F}_{2.5}$ as the low activity of solvent molecules, however, due to the unstable interface, the specific capacity is continuously attenuated and the polarization is gradually increased. Sulfur content $=50 \%$. 


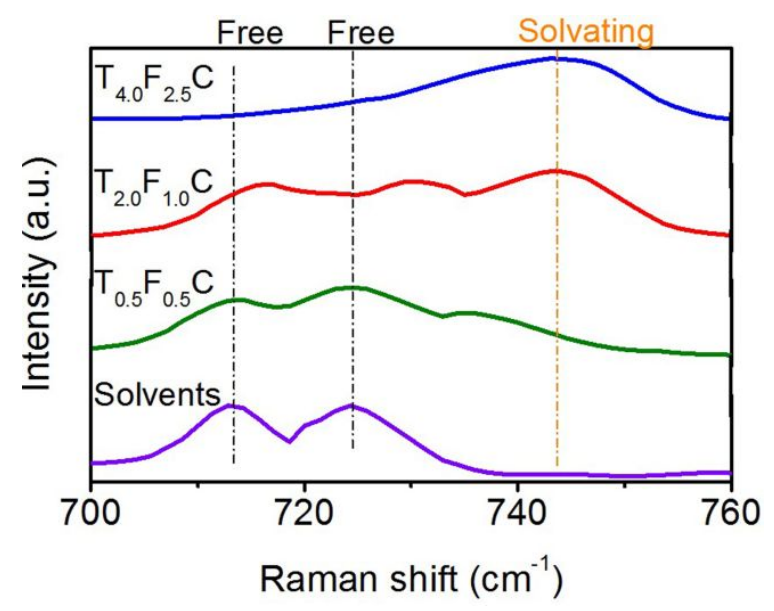

Figure S7. Raman spectra of LiTFSI-LiFSI/EC:DEC:FEC solutions in a) $700-760 \mathrm{~cm}^{-1}$ (the peak position local at 713 and $725 \mathrm{~cm}^{-1}$ is assigned to symmetric ring deformation mode of EC and FEC, $736 \mathrm{~cm}^{-1}$ is S-N stretching mode of TFSI-, $744 \mathrm{~cm}^{-1}$ is attributed to solvated solvent molecules and anions. At a concentration of $\mathrm{T}_{4.0} \mathrm{~F}_{2.5} \mathrm{C}$, there is no free solvent molecule and solvated anions of TFSI- ${ }^{-}$and FSI-shift to right). ${ }^{1-3}$
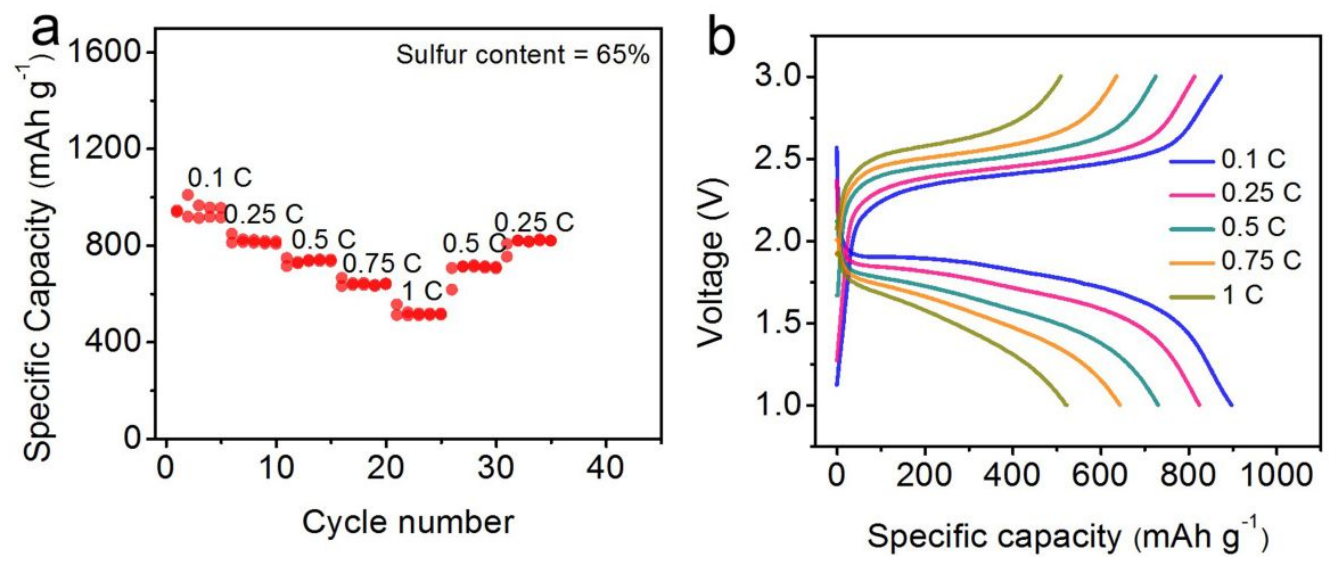

Figure S8. The CMK-3/S@SEI in $\mathrm{T}_{4.0} \mathrm{~F}_{2.5} \mathrm{C}$ electrolyte; a) rate performance, b) the charge/discharge curves at various rates. 

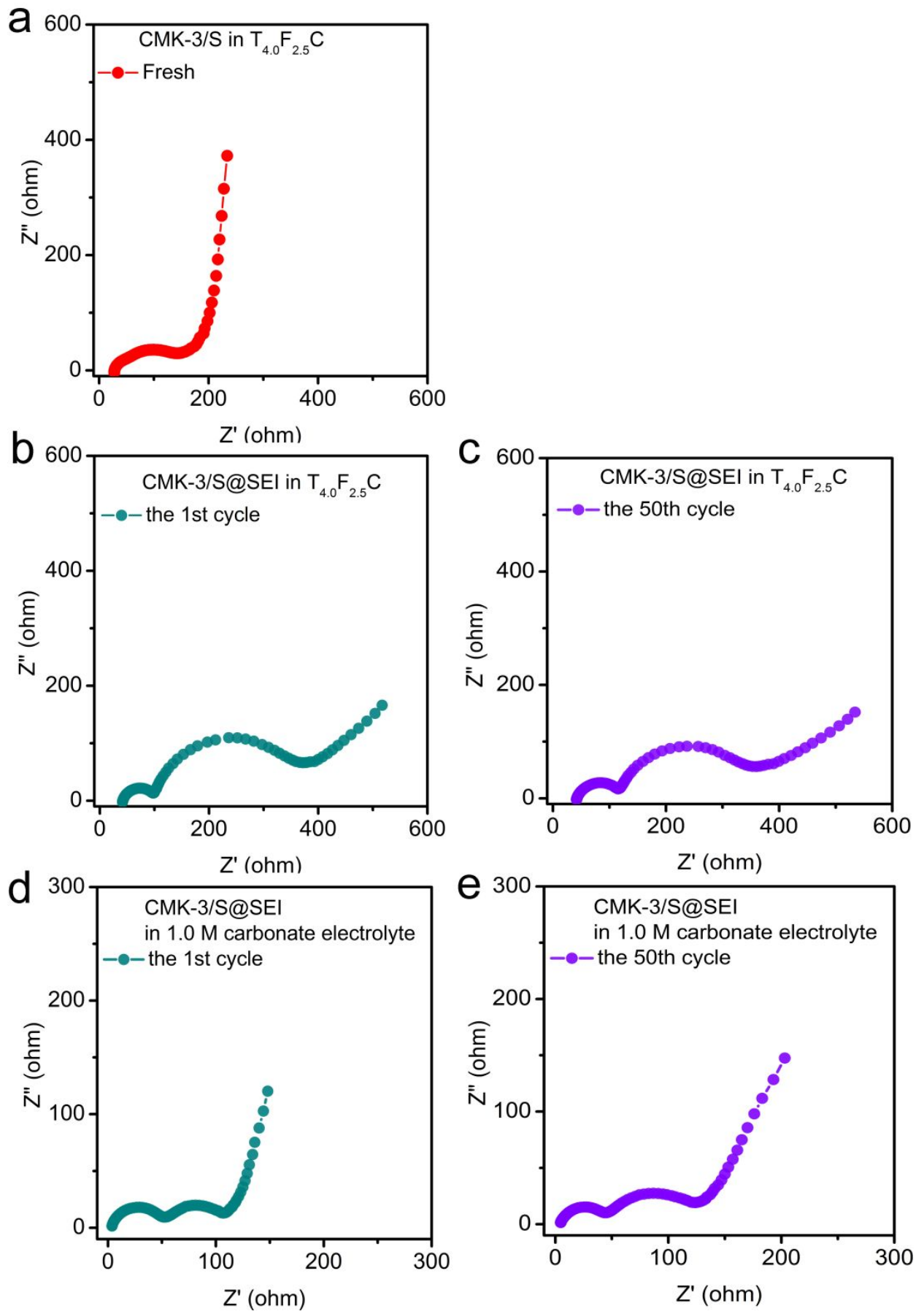

Figure S9. a) The EIS spectra of a fresh CMK-3/S cathode in $\mathrm{T}_{4.0} \mathrm{~F}_{2.5} \mathrm{C}$. The EIS spectra of CMK-3/S@SEI cathode at $1.0 \mathrm{~V}$ in b) the 1 st and c) the 50th cycles in the $\mathrm{T}_{4.0} \mathrm{~F}_{2.5} \mathrm{C}$ electrolyte. The EIS spectra of CMK-3/S@SEI at $1.0 \mathrm{~V}$ in d) the 1st and e) the 50th cycles in the $1.0 \mathrm{M}$ carbonate electrolyte. 

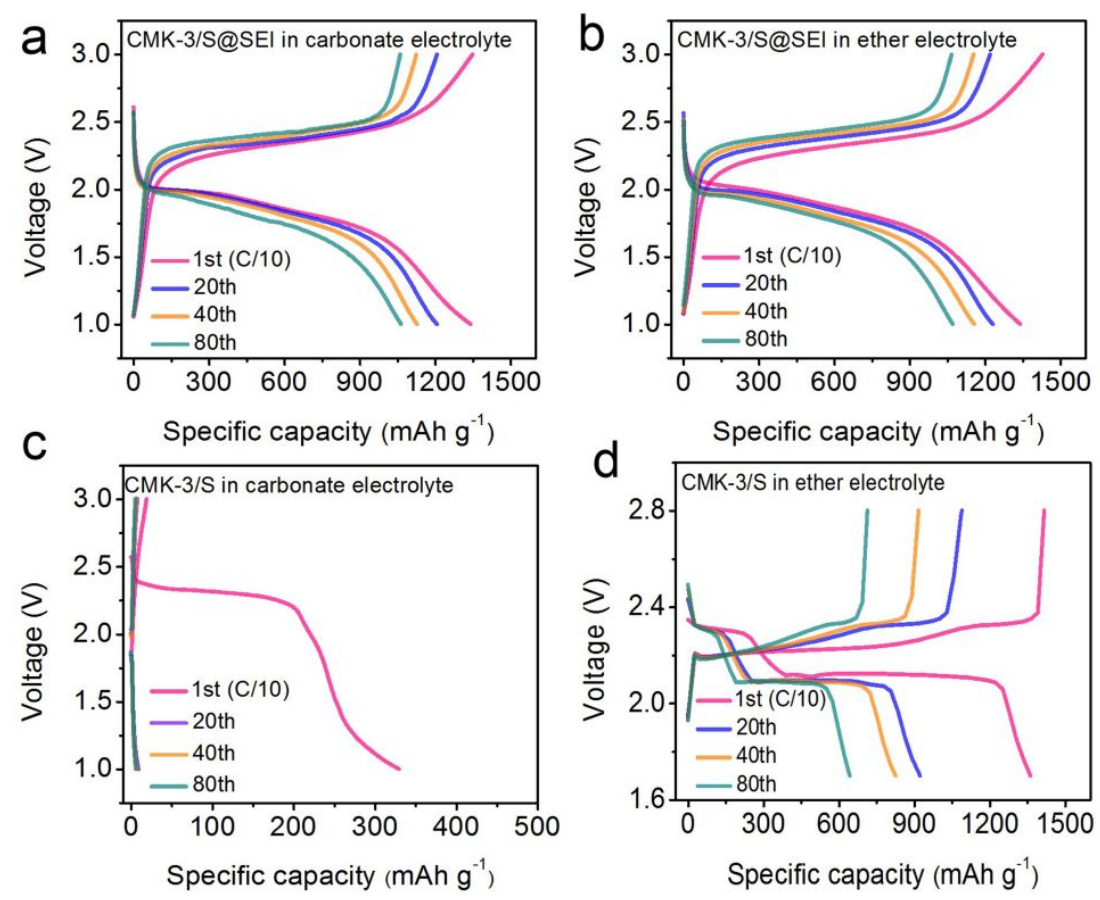

Figure S10. The charge/discharge curves of CMK-3/S@SEI in: a) $1.0 \mathrm{M}$ carbonate electrolyte, b) 1.0 M ether electrolyte. The charge-discharge curves of CMK-3/S in: c) $1.0 \mathrm{M}$ carbonate electrolyte, d) $1.0 \mathrm{M}$ ether electrolyte. Sulfur content $=50 \%$.

\section{REFERENCES}

(1) Huang, L.-Y.; Shih, Y.-C.; Wang, S.-H.; Kuo, P.-L.; Teng, H. Gel Electrolytes Based on an Ether-Abundant Polymeric Framework for High-Rate and Long-Cycle-life Lithium Ion Batteries. J. Mater. Chem. A 2014, 2, 10492-10501.

(2) Yamanaka, T.; Nakagawa, H.; Tsubouchi, S.; Domi, Y.; Doi, T.; Abe, T.; Ogumi, Z. In Situ Raman Spectroscopic Studies on Concentration of Electrolyte Salt in Lithium-Ion Batteries by Using Ultrafine Multifiber Probes. ChemSusChem 2017, 10, 855-861. 
(3) Yamada, Y.; Takazawa, Y.; Miyazaki, K.; Abe, T. Electrochemical Lithium Intercalation into Graphite in Dimethyl Sulfoxide-Based Electrolytes: Effect of Solvation Structure of Lithium Ion. J. Phys. Chem. C 2010, 114, 11680-11685. 